\title{
Long-period Fiber Grating Effects with Double-sided Loading on Fiber
}

\author{
Chung-Yih Tang ${ }^{1}$, Paoyi Tseng ${ }^{2}$, Chih-Nan Lin $^{3}$, Chih-Chung (C. C.) Yang ${ }^{1,2,4}$, Yean-Woei Kiang ${ }^{1,2,3}$ and \\ Kung-Jen $\mathrm{Ma}^{5}$ \\ ${ }^{1}$ Graduate Institute of Electro-Optical Engineering, National Taiwan University, \\ No. 1, Roosevelt Road, Sec. 4, Taipei, Taiwan \\ (phone) 886-2-23657624 (fax) 886-2-23652637 (E-mail) ccy@cc.ee.ntu.edu.tw \\ ${ }^{2}$ Department of Electrical Engineering, National Taiwan University, Taipei, Taiwan \\ ${ }^{3}$ Graduate Institute of Communication Engineering, National Taiwan University, Taipei, Taiwan \\ ${ }^{4}$ Graduate Institute of Electronics Engineering, National Taiwan University, Taipei, Taiwan \\ ${ }^{5}$ Department of Mechanical Engineering, Chung Hua University, Hsinchu, Taiwan
}

\begin{abstract}
Periodical perturbations along an optical fiber can cause power coupling between the core mode and cladding modes for the applications of spectral filtering and derivative operations. Such perturbations can be generated through periodical loading on fiber. By applying loading onto fiber with two face-to-face, identical periodical groove structures, it was found that the long-period grating effects were dependent on the relative phase of the two periodical corrugations. Particularly, when the relative phase was zero (crest-to-crest), spectral filtering effects disappeared completely. The comparisons of such results between the cases of jacketed and un-jacketed fibers led to the conclusion that geometric deformation, instead of direct pressure-induced effect, was the dominating mechanism for generating spectral filtering functions in the double-sided loading configuration. The same conclusion can be applied to the single-sided loading device.
\end{abstract}

Keywords: long-period fiber grating, mode coupling, micro bending

\section{INTRODUCTION}

Spectral filtering and its derivative operations, such as gain flattening [e.g., 1] are important functions for fiber communication applications. Spectral filtering can be implemented with long-period fiber gratings of various forms. With a long-period fiber grating for phase matching, the core-mode signal in the fiber can be coupled into cladding modes, resulting in spectral depressions. Effective long-period fiber gratings can be realized with either permanent modification of fiber, such as etching [2] and UV-induced refractive index change [3], or temporary alteration of fiber propagation characteristics. The method of temporary alteration has the advantage of dynamically controlling the function of spectral filtering. Dynamic long-period fiber gratings can be implemented through the application of either an acoustic wave [4]-[7], or periodical loading onto fiber [8]-[10]. In the loading technique, two possible mechanisms may result in the aforementioned phase matching process, including geometric deformation (micro-bending) and pressure effect for periodical refractive index variation of the fiber. In this letter, we report the spectral filtering results of effective long-period gratings implemented by double-sided loading with two identical blocks of periodical grooves. Also, the dominating mechanism for effective grating effects is discussed. In our experiments, it was found that the positions and depths of transmission spectral depressions varied with the relative phase of the two periodically grooved structures. When the two periodical structures are exactly in phase, i.e., crests exactly matched, all spectral depressions disappear. This is true no matter the plastic jacket is removed or not. This observation leads to the conclusion that geometric deformation is the dominating mechanism for phase matching between the core and cladding modes. The conclusion is further confirmed with the results of single-sided loading on fiber, which manifests that loading without plastic jacket is less effective than the case with jacket in the loading range that fiber is not broken. The jacket is supposed to provide the means for micro bending, which results in effective mode coupling.

\section{EXPERIMENTAL SETUP AND RESULTS}

For double-sided loading, two identical copper blocks of $3 \times 3 \times 0.5 \mathrm{~cm}(\mathrm{~L} \mathrm{x} \mathrm{W} \mathrm{x} \mathrm{T)} \mathrm{with} \mathrm{periodical} \mathrm{corrugations} \mathrm{on} \mathrm{the} \mathrm{L} \times$ W plane were prepared. Square grooves of $400 \mu \mathrm{m}$ in depth and of $50 \%$ in duty cycle were fabricated with mechanical process. Copper blocks with various corrugation periods between 500 and $700 \mu \mathrm{m}$ were prepared. Commonly used communication fiber (Corning, SMF-28) was placed between the two corrugated copper blocks. Loading was applied by moving one of the blocks toward the other with a translation stage. The relative position, i.e., relative phase, of the two 
face-to-face corrugated blocks was controlled by a translation stage moving along another direction. The fiber was aligned along the normal to the groove lines. The transmission spectrum of the fiber under loading was recorded with an HP spectrum analyzer with spectral resolution of $0.08 \mathrm{~nm}$.

Figure 1 shows two normalized transmission spectra when the $700 \mu \mathrm{m}$ corrugation set was used for two loading levels: 0.15 and $0.2 \mathrm{~mm}$ stage displacements for the two curves from top to bottom, respectively. In this situation, the relative phase of the two blocks was about $\pi / 2$. Note that zero-phase is defined as the situation when crests of the two corrugated blocks are exactly matched. The spectra shown in Fig. 1 were normalized to the spectral level of zero loading (completely loose). Three depressions around 1500, 1560 and $1620 \mathrm{~nm}$ can be clearly seen. The deeper depressions are due to stronger coupling between the core mode and various cladding modes.

The wavelength and depth of the minimums of similar depressions were recorded when the relative phase of the two corrugated blocks were varied. Figures 2 and 3 show the variations of depression wavelength and depth with the relative phase when the loading level is about $0.22 \mathrm{~mm}$ stage displacement and the corrugation period is $700 \mu \mathrm{m}$. Here, the relative phase is also represented with the stage displacement in mm. In either Fig. 2 or 3, periodical variations with period $700 \mu \mathrm{m}$ can be clearly seen, confirming the $700-\mu \mathrm{m}$ corrugation period. Only two coupling wavelengths are shown because the one near $1500 \mathrm{~nm}$ is unclear (see Fig. 1). The other abscissa in either Fig. 2 or 3 indicates the corresponding relative phase. One can clearly see that near the phases of integers of $2 \pi$, all the induced spectral depressions disappear. Figure 4 shows the comparison of spectra between the cases without loading (the lower curve) and with loading (the upper curve, with $0.22 \mathrm{~mm}$ stage displacement loading level - a relatively high loading level) when the relative phase is an integer of $2 \pi$. We can see the almost identical spectra without any significant spectral depression (even without a significant difference in background level). The ratio of the two curves results in $<7 \%$ noise fluctuation within the spectral range from 1.4 through $1.7 \mu \mathrm{m}$. Such results confirm the insignificant effect of loading when the relative phase is an integer of $2 \pi$. This conclusion is also true for other corrugation periods.

Fig. 2 shows the wavelength variations of depression locations. It is noted that the depression wavelengths reach the longest values when the relative phase is an odd integer of $\pi$. In this situation, the signal coupling is expected to be strongest because the depression depth increases as the relative phase moves away from integers of $2 \pi$, as shown in Fig. 3 . The tuning range in varying the relative phase is about $10 \mathrm{~nm}$. Such wavelength variations can be attributed to the change of average refractive index in loading with different relative corrugation phases. As shown in Fig. 4, the spectral depression depth drops fast when the relative phase approaches an integer of $2 \pi$. The minimum features of depression depth near odd numbers of $\pi$ relative phase may origin from the saturation effects of loading. Note that the non-smooth data point variations in Figs. 2 and 3 can be attributed to the imperfect periodical grooves on copper blocks.

The insignificant loading effect in the case that the relative phase is an integer of $2 \pi$ has an important implication. Application of loading onto fiber may produce two mechanisms for inducing mode coupling: geometric deformation and pressure-induced elasto-optical effect. The former forms micro bending for phase matching. The later generates periodical refractive index variation for phase matching. From the results described above, we may conclude that geometric deformation is the dominating mechanism for phase matching in mode coupling in our loading configuration. This conclusion can be understood with Figs. 5(a) and (b). In Fig. 5(a), the loading effect on fiber with zero relative-phase is schematically depicted. Here, one can see that the loading effect is essentially the periodical application of pressure onto fiber. Such a pressure may not be effectively applied to the glass portion of the fiber because the plastic jacket may absorb the pressure. On the other hand, as shown in Fig. 5(b) the loading effect on fiber with $\pi$ phase is essentially geometric deformation, i.e., formation of micro bending. Such a geometric deformation produces significant coupling between the core mode and cladding modes, as shown in Figs. 2 and 3. When the relative phase moves from $\pi$ to zero, the micro bending amplitude is reduced and the effective grating period is decreased. Therefore, the coupling strength, i.e., spectral depression depth, is reduced, as shown in Fig. 3. Also, the depression wavelength becomes shorter when the relative phase moves from $\pi$ to zero, as shown in Fig. 2 .

As mentioned, one may wonder that the insignificant loading effect in the case of zero relative-phase may origin from the pressure absorption of fiber jacket. To understand the jacket effects, we removed the jacket and conducted the loading experiments. We observed almost the same results, except that the loading level could not be too large, otherwise the fiber was broken. Particularly, again insignificant loading effect could be observed in the case of zero relative-phase. The transmission spectra of the un-loaded and loaded cases look almost identical.

\section{DISCUSSIONS}

The observations described so far may cause a doubt about the effects of single-sided loading [10]. By intuition, single-sided loading cannot create micro bending of fiber and its grating effects must come from periodical pressure distribution. To clarify this point, we also conducted single-sided loading with jacketed and un-jacketed fibers. Figure 6 shows the loading results with the solid curve for the case with jacket and the dashed curve for the case without jacket. We did observe the grating effects with single-sided loading, either with jacket or without jacket. However, the loading effect with jacket is much 
more significant. The results in Fig. 6 rule out the possible effect of periodical pressure in loading because the existence of jacket, which is supposed to absorb pressure, resulted in much stronger loading effect. Such a result can only be explained with the conclusion that even in the case of single-sided loading, the dominating mechanism for mode coupling is micro bending. As depicted in Fig. 5(c), the elasticity of jacket can help the fiber to become curved when the corrugated loading is applied. When the jacket is removed, it becomes difficult to produce micro bending along rigid glass.

\section{CONCLUSIONS}

In summary, we have demonstrated the spectral filtering effects of fiber by double-sided loading with identical periodical corrugations. It was found that the filtering wavelength and strength were dependent on the relative phase of the two periodical corrugations. Particularly, when the relative phase was zero (crest-to-crest), spectral filtering effects disappeared completely. Such results and other evidences led to the conclusion that geometric deformation, instead of direct pressure-induced effect, was the dominating mechanism for generating spectral filtering functions in the double-sided loading configuration. Such a conclusion should also be true for the configuration of single-sided loading.

\section{ACKNOWLEDGEMENTS}

This research was supported by National Science Council, The Republic of China, under the grants of NSC 89-2218-E-002-094, NSC 89-2218-E-002-095, and NSC 89-2215-E-002-051.

\section{REFERENCES}

[1] M. Tachibana, R. I. Laming, P. R. Morkel, and D. N. Payne, "Erbium-doped fiber amplifier with flattened gain spectrum," IEEE Photo. Tech. Lett., Vol. 3, pp. 118-120, 1991.

[2] S. A. Vasiliev, E. M. Dianov, D. Varelas, H. G. Limberger, and R. P. Salathe, "Postfabrication resonance peak positioning of long-period cladding-mode-coupled gratings," Opt. Lett., Vol. 21, pp. 1830-1832, 1996.

[3] A. M. Vengsarkar, P. J. Lemaire, J. B. Judkins, V. Bhatia, T. Erdogan, and J. E. Sipe, "Long-period fiber gratings as band-rejection filter," J. Lightwave Tech., Vol. 14, pp. 58-65, 1996.

[4] R. Faced, C. Alegria, M. N. Zervas, and R. I. Laming, "Acoustooptic attenuation filters based on tapered optical fibers," IEEE J. Select. Topic in Quantum Electron., Vol. 5, pp. 1278-1288, 1999.

[5] D. W. Huang, W. F. Liu, C. W, Wu and C. C. Yang, "Reflectivity-tunable fiber Bragg grating reflectors," IEEE Photon. Tech. Lett., Vol. 12, pp. 176-178, 2000.

[6] W. F. Liu, I. M. Liu, L. W. Chung, D. W. Huang, and C. C. Yang, "Acoustics-induced reflection wavelength switching in a fiber Bragg grating," Opt. Lett., Vol. 25, pp. 1319-1321, 2000.

[7] A. Diez, T. A. Birks, W. H. Reeves, B. J. Mangan, and P. St. J. Russell, "Excitation of cladding modes in photonic crystal fibers by flexural acoustic waves," Opt. Lett., Vol. 25, pp. 1499-1501, 2000.

[8] R. C. Youngquist, J. L. Brooks, and H. J. Shaw, "Birefringent-fiber polarization coupler," Opt. Lett., Vol. 8, pp. 656-658, 1983.

[9] R. C. Youngquist, J. L. Brooks, and H. J. Shaw, "Two-mode fiber modal coupler," Opt. Lett., Vol. 9, pp. 177-179, 1984.

[10] S. Savin, M. J. F. Digonnet, G. S. Kino, and H. J. Shaw, "Tunable mechanically induced long-period fiebr gratings," Opt. Lett., Vol. 25, pp. 710-712, 2000.

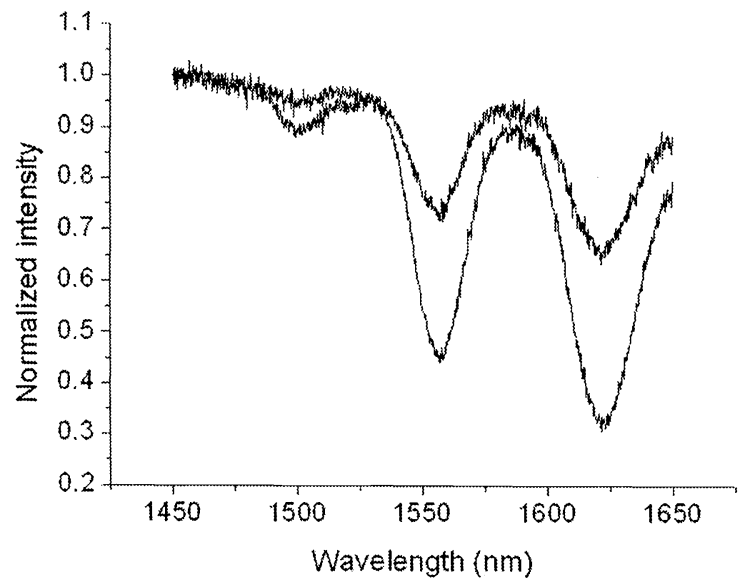

Fig. 1 Typical normalized transmission spectrum of double-side loaded fiber grating with corrugation period of $700 \mu \mathrm{m}$. The loading displacements for the two curves are 0.15 and $0.2 \mathrm{~mm}$. The relative phase of the two periodical corrugations is about $\pi / 2$. 


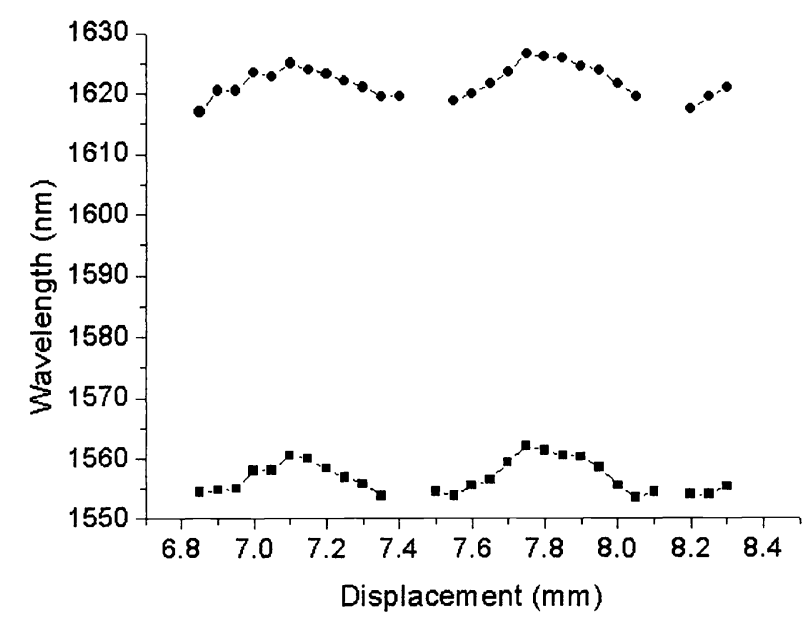

Fig. 2 Variations of the wavelengths of spectral depression minimums with the relative phase of the two periodical corrugations when the loading level is $0.22 \mathrm{~mm}$.

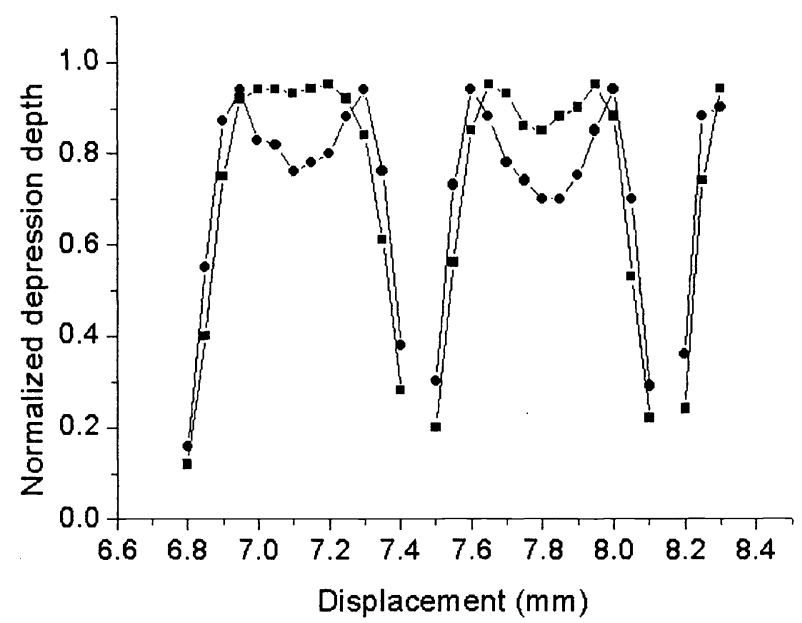

Fig. 3 Variations of the depths of spectral depression minimums with the relative phase of the two periodical corrugations.

The two data sets correspond to those of Fig. 2.

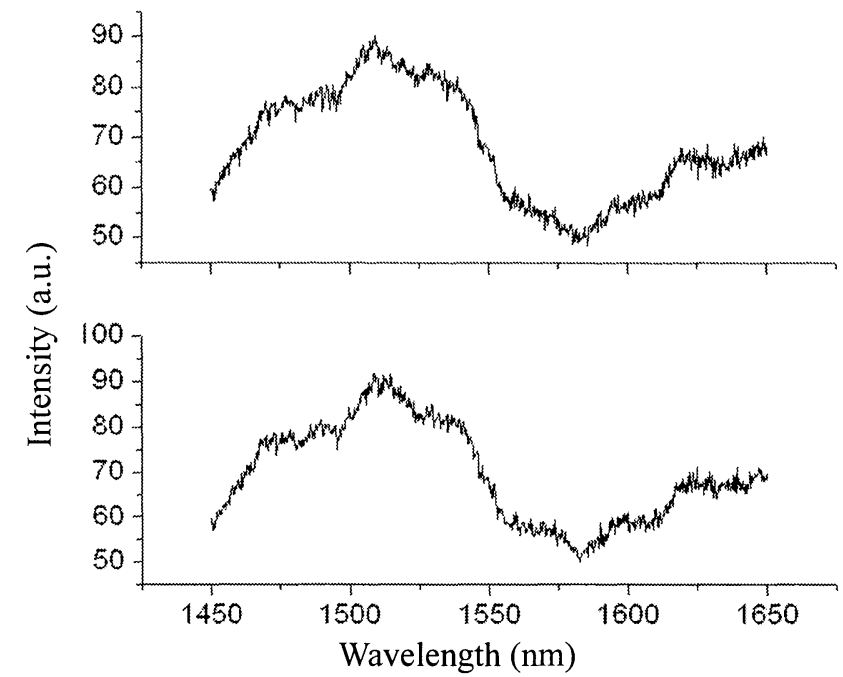

Fig. 4 Transmission spectra of the case with zero relative phase for unloaded (the lower curve) and loading with $0.22 \mathrm{~mm}$ displacement loading (the upper curve) conditions. The two spectra are almost identical. 
(a)
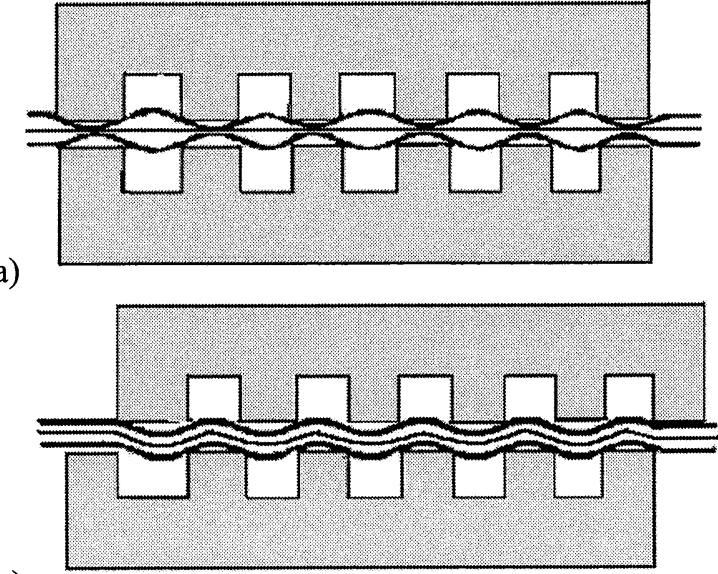

(b)

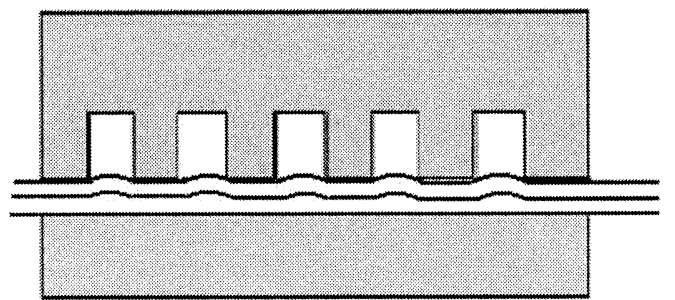

(c)

Fig. 5 Schematic drawings of fiber deformations in the cases of zero (a) and $\pi$ (b) relative phases of the two periodical corrugations in double-sided loading and in the case of single-sided loading (c). Micro bending is expected in the cases of (b) and (c).

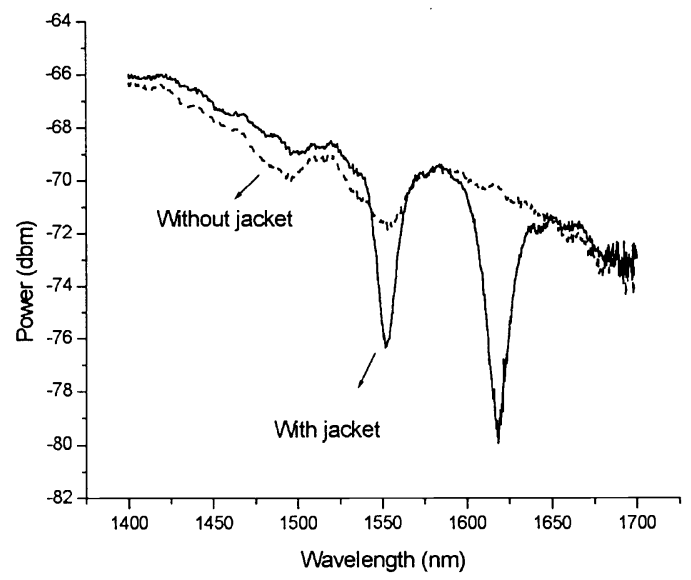

Fig. 6 Transmission spectra of single-sided loaded fiber in the cases of keeping jacket (solid curve) and removing jacket (dashed curve). 